\title{
Use of lopinavir/ritonavir in first-line therapy or second-line therapy: 48-week results from the German prospective STAR cohort

\author{
C Koegl*1, A Trein ${ }^{2}$, W Schmidt ${ }^{3}$, A Baumgarten ${ }^{4}$, H Jaeger ${ }^{5}$ and \\ HJ Stellbrink 6
}

Address: ${ }^{1}$ MUC Reseach, Munich, Germany, ${ }^{2}$ HIV Center, Stuttgart, Germany, ${ }^{3}$ Aerzteforum Seestraße, Berlin, Germany, ${ }^{4}$ HIV-Practice Berlin Prenzlauerberg, Berlin, Germany, ${ }^{5} \mathrm{HIV}$ Research and Clinical Care Centre, Munich, Germany and ${ }^{6} \mathrm{ICH}$ (Infektionsmedizinisches Zentrum Hamburg), Hamburg, Germany

* Corresponding author

from Ninth International Congress on Drug Therapy in HIV Infection

Glasgow, UK. 9-13 November 2008

Published: 10 November 2008

Journal of the International AIDS Society 2008, I I (SuppI I):P75 doi:I0.I I86/I758-2652-II-SI-P75

This abstract is available from: http://www.jiasociety.org/content/II/SI/P75

(C) 2008 Koegl et al; licensee BioMed Central Ltd.

\section{Purpose of the study}

Treatment guidelines are mainly based on the evidence of efficacy in clinical trials. However, treatment outcomes may differ in observational cohort studies. We analysed data from the STAR cohort, a German prospective, multicentre, observational study, which includes HIV+ patients (pts) starting a lopinavir/ritonavir (LPV/r)-based regimen. Our goal was to compare treatment outcomes in ARTnaïve pts compared to pre-treated, but PI-naïve pts.

\section{Methods}

48-week analysis: comparison of treatment responses to LPV/r-based regimens in ART-naïve and PI-naïve pts using on-treatment (OT), intent-to-treat (ITT) and KaplanMeier (KM) analyses. Primary outcome parameters were reaching a confirmed viral load (VL) $<50$ copies $/ \mathrm{ml}, \%$ with $\mathrm{VL}<50$ copies $/ \mathrm{ml}$, and time to reaching a confirmed CD4 count $>500 / \mu \mathrm{l}$.

\section{Summary of results}

1,079 HIV+ pts (74\% ART-naive) have been included in the STAR cohort. Median age was 41 years (range: $20-76$ ). Currently, baseline values were available of 872 pts (76\% [662] ART-naïve). Median baseline VL and CD4 cell counts differed significantly between ART-naïve and PInaïve pts $(125,500$ vs. 3,746 copies $/ \mathrm{ml}, \mathrm{p}<0.001 ; 198$ vs. $310 / \mu \mathrm{l}, \mathrm{p}<0.001)$.
At 48 weeks, VL was $<50$ copies $/ \mathrm{ml}$ in $79 \%$ of ART-naive and in $75 \%$ of PI-naive pts (OT) (ITT: 62\% of ART-naive and $60 \%$ of PI-naive pts). Median changes in CD4 cells were $+201 / \mu \mathrm{l}$ in ART-naïve and $+135 / \mu \mathrm{l}$ in PI-naïve pts (p $<0.001)$. In $60 \%$ of ART-naive pts, CD4 cells increased to levels $>350 / \mu \mathrm{l}$ and in $34 \%$ to levels $>500 / \mu \mathrm{l}$. In $73 \%$ of PInaive pts, CD4 cells increased to levels $>350 / \mu \mathrm{l}$ and in $48 \%$ to levels $>500 / \mu \mathrm{l}$.

The probability of remaining of treatment was 78 vs. $77 \%$ in ART-naïve vs. PI-naïve pts at 48 weeks (KM-analysis, $\mathrm{p}$ $=\mathrm{ns}$ ). The median time to confirmed $\mathrm{VL}<50$ copies $/ \mathrm{ml}$ was 25 weeks in ART-naive and 13 weeks in PI-naïve pts; time to $>500 \mathrm{CD} 4$ cells/ $\mu \mathrm{l}$ was 73 weeks in ART-naïve and 48 weeks in PI-naive pts.

\section{Conclusion}

In this cohort study, virological outcomes of LPV/r-based regimens were comparable in ART-naïve and in pretreated PI-naïve pts, reflecting good efficacy and durability also in second- or third-line use of LPV/r. The probability of remaining on treatment for 48 weeks was high in both groups (78 and $77 \%$ ); VL was $<50$ copies $/ \mathrm{ml}$ in $79 \%$ of ART-naive and in $75 \%$ of PI-naive pts (OT) (ITT: $62 \%$ and $60 \%$ ). In $34 \%$ of ART-naive pts and in $48 \%$ of PI-naïve pts, CD4 cells increased to levels $>500 / \mu \mathrm{l}$, demonstrating an ongoing CD4 increase also in pre-treated PI-naïve pts. 